\title{
气相 $\mathrm{Cr}_{x} \mathrm{~N}^{-}(x=2 \sim 7)$ 阴离子团簇与 $\mathrm{CO}_{2}$ 的反应研究
}

\author{
王明 ${ }^{\dagger}$, 周海岩 ${ }^{\dagger}$, 崔佳桐, 孙传新, 马嘉璧 \\ 北京理工大学化学与化工学院, 原子分子簇科学教育部重点实验室, 光电转换材料北京市重点实验室, 北京 102488 \\ †共同贡献 \\ *通讯作者, E-mail: majiabi@bit.edu.cn
}

收稿日期：2020-04-30; 接受日期：2020-07-07; 网络版发表日期：2020-08-24

国家重点研发计划(编号: 2016YFC0203000)、国家自然科学基金(编号: 21503015)和中央高校基本科研业务费专项资金(编号: 2017CX01008) 资助项目

\begin{abstract}
摘要室温下将二氧化碳 $\left(\mathrm{CO}_{2}\right)$ 高效还原为一氧化碳 $(\mathrm{CO})$ 对于 $\mathrm{CO}_{2}$ 资源的利用至关重要. 本文通过高分辨率反 射式飞行时间质谱结合密度泛函理论(DFT)计算，在热碰撞条件下利用铬氮阴离子团簇 $\mathrm{Cr}_{x} \mathrm{~N}^{-}(x=2 \sim 7)$ 实现了连 续还原两个 $\mathrm{CO}_{2}$ 分子. $\mathrm{Cr}_{x} \mathrm{~N}^{-}(x=2 \sim 7)$ 和 $\mathrm{Cr}_{2} \mathrm{NH}^{-}$团簇可以与 $\mathrm{CO}_{2}$ 反应分别生成 $\mathrm{Cr}_{x} \mathrm{NO}^{-} / \mathrm{CO}$ 和 $\mathrm{Cr}_{2} \mathrm{NHO}^{-} / \mathrm{CO}$, 之后中 间产物三元阴离子 $\mathrm{Cr}_{x} \mathrm{NO}^{-}$可以进一步还原第二个 $\mathrm{CO}_{2}$ 生成 $\mathrm{Cr}_{x} \mathrm{NO}_{2}{ }^{-}$和 $\mathrm{CO}$ 分子. 上述连续反应具有高反应活性, 并 且无 $\mathrm{CO}_{2}$ 吸附产物生成. 随着团簇中铬原子数目从2增加到 $7\left(\mathrm{Cr}_{2} \mathrm{~N}^{-} \sim \mathrm{Cr}_{7} \mathrm{~N}^{-}\right)$, 团簇的反应活性先增加后略有降低. DFT计算给出了该类活性阴离子的结构和连续反应的反应机理。这项工作为逆水煤气催化剂表面活性位的设计 提供了新的思路.
\end{abstract}

关键词气相反应, 二氧化碳, 过渡金属氮化物团簇, 质谱, 量子化学计算

\section{1 引言}

二氧化碳 $\left(\mathrm{CO}_{2}\right)$ 的还原反应不仅可以缓解大气温 室效应，而且可以提供将废物转化为燃料和工业化学 品的方法 ${ }^{[1,2]}$, 所以近年来一直受到研究人员的广泛关 注. 在利用 $\mathrm{H}_{2}$ 还原 $\mathrm{CO}_{2}$ 过程中, 存在两种共存的反应途 径: (1) 逆水煤气反应(the reverse water gas shift, $\mathrm{RWGS}$ ), 即 $\mathrm{CO}_{2}+\mathrm{H}_{2} \rightarrow \mathrm{CO}+\mathrm{H}_{2} \mathrm{O}$; (2) 甲烷化反应(methanation reaction), 即 $\mathrm{CO}_{2}+4 \mathrm{H}_{2} \rightarrow \mathrm{CH}_{4}+2 \mathrm{H}_{2} \mathrm{O}$. 为了实现对 产物 $\mathrm{CO}$ 和 $\mathrm{CH}_{4}$ 的高效选择性催化, 研究人员进行了大 量研究 ${ }^{[3 \sim 5]}$, 其中利用RWGS反应将 $\mathrm{CO}_{2}$ 催化转化为 $\mathrm{CO}$ 被认为是很有前途的 $\mathrm{CO}_{2}$ 转化工艺之一. 在上述提到
的RWGS和甲烷化反应中，人们普遍认为吸附态 $\mathrm{CO}$ $\left(\mathrm{CO}_{\mathrm{ad}}\right)$ 是一个关键中间体 ${ }^{[3,4]}$. 目前, 关于 $\mathrm{CO}_{\mathrm{ad}}$ 的形成 机制仍存在争论: 一种观点认为 $\mathrm{CO}_{\mathrm{ad}}$ 是通过RWGS 反 应生成甲酸盐中间体形成的 ${ }^{[6]}$; 另一种观点认为 $\mathrm{CO}_{\mathrm{ad}}$ 是通过活性金属中心的氧化还原机理形成的 $\left(x \mathrm{CO}_{2}+\right.$ $\left.\mathrm{M}^{n+} \rightarrow \mathrm{MO}_{x}+x \mathrm{CO}\right)^{[7]}$. 在已报道的 $\mathrm{CO}_{2}$ 还原生成 $\mathrm{CO}$ 的反 应中, 负载金属氧化物的催化剂是主要的研究对象, 过渡金属碳化物也是RWGS催化剂的潜在载体 ${ }^{[8]}$. 然 而, 关于负载过渡金属氮化物的催化剂的研究却很少. 值得注意的是, 由于 $\mathrm{CO}_{2}$ 热力学和动力学的稳定性, 在 非均相催化转化反应中, 往往需要较高的催化剂床层 温度. 在温和条件下, $\mathrm{CO}_{2}$ 的催化活化和转化仍具有相

引用格式: Wang M, Zhou H, Cui J, Sun C, Ma J. The study on the reaction of gas-phase $\mathrm{Cr}_{x} \mathrm{~N}^{-}(x=2-7)$ anion clusters with $\mathrm{CO}_{2}$. Sci Sin Chim, 2020, 50: 11691176, doi: 10.1360/SSC-2020-0061 
当大的挑战. 了解催化剂表面活性位点的内在性质和 反应机理(如将 $\mathrm{CO}_{2}$ 有效还原为 $\mathrm{CO}$ )非常关键.

通过实验与计算方法相结合对“孤立的”反应物 进行气相反应研究, 可以为从分子水平揭示反应机理 和动力学提供一个理想的场所 ${ }^{[9 \sim 23]}$. 气相离子与 $\mathrm{CO}_{2}$ 的反应已在几篇优秀的综述论文中进行了详细讨 论 ${ }^{[9,24]}$, 可以总结为三个基本反应, 即(1) $\mathrm{CO}_{2}$ 还原成 $\mathrm{CO}$; (2) 将氢化物转移到 $\mathrm{CO}_{2}$ 生成甲酸盐; (3) 偶联反 应生成 $\mathrm{C}-\mathrm{X}$ 键 ${ }^{[11]}$. 在过去的十几年中，一些阳离子与 $\mathrm{CO}_{2}$ 的反应被相继报道 ${ }^{25 \sim 45]}$, 其中大多数体系是作为 氧受体的金属氧化物团簇，而其他离子则具有各种配 体, 如环戊二烯络合物 $\left[\mathrm{Cp}_{2} \mathrm{TiH}^{+}\right]^{[45]}$. 除这些离子外, 中 性的 $\mathrm{TiO}$ 和 $\mathrm{TiO}_{2}$ 分子对 $\mathrm{CO}_{2}$ 也有活性 $\left(\mathrm{TiO}+\mathrm{CO}_{2} \rightarrow \mathrm{TiO}_{2}\right.$ $+\mathrm{CO}$ 和 $\left.\mathrm{TiO}_{2}+\mathrm{CO}_{2} \rightarrow \mathrm{TiO}_{2} \mathrm{CO}_{2}\right)^{[46]}$. 与大多数研究的阳离 子体系相比，带负电荷的阴离子与 $\mathrm{CO}_{2}$ 的反应报道则 比较少. 最近有研究表明, $\mathrm{FeH}^{-}$和 $\mathrm{Re}(\mathrm{CO})_{2}{ }^{+}$均可与 $\mathrm{CO}_{2}$ 反应, 分别生成 $\mathrm{CO}_{2} \mathrm{H}^{-[47]}$ 以及通过氧原子转移 (oxygen-atom transfer, OAT)生成 $\operatorname{Re}(\mathrm{CO})_{2} \mathrm{O}^{+[43]}$. 在上 述反应中, 气相离子中的金属原子对 $\mathrm{CO}_{2}$ 的活化起重 要作用.

在本文中，我们报道了关于 $\mathrm{Cr}_{x} \mathrm{~N}^{-} \quad(x=2 \sim 7)$ 和 $\mathrm{Cr}_{2} \mathrm{NH}^{-}$阴离子团簇与 $\mathrm{CO}_{2}$ 反应的研究. 该研究是气相 团簇化学领域中，关于多核过渡金属氮化物团簇阴离 子活化转化 $\mathrm{CO}_{2}$ 的第一个例子.

\section{2 实验部分}

\section{1 实验方法}

实验仪器主要采用四极杆选质-线性离子阱反应 池-耦合高分辨率反射式飞行时间质谱(time-of-flight mass spectrometer, TOF-MS)检测离子团簇与 $\mathrm{CO}_{2}$ 分子 的反应, 该质谱仪配备有激光溅射离子源 (ion source)、四极杆质量过滤器(quadrupole mass filter, QMF)和线性离子阱(linear ion trap, LIT)反应器, 仪器 部分在之前的工作已详细描述 ${ }^{[48 \sim 50]}$, 下面仅给出实验 方法的简要概述. $\mathrm{Cr}_{x} \mathrm{~N}^{-}(x=2 \sim 7)$ 阴离子团簇是通过一 束脉冲激光溅射到螺旋转动的铬靶(纯度: $99.999 \%$ )上, 并在产生管中与氨气(浓度 $0.02 \%$, 背景气为 $\mathrm{He}$ 气，背 景压力为 $4 \mathrm{~atm}$ )碰撞、反应、冷却聚集而产生. 所用 脉冲激光为 $532 \mathrm{~nm}$ 波长 $\left(\mathrm{Nd}^{3+}\right.$ 的二次谐波: 固体脉冲激 光器的二倍频), 使用能量为 $5 \sim 8 \mathrm{~mJ} /$ 脉冲, 工作频率为
$10 \mathrm{~Hz}$. 团簇离子经产生管超声膨胀冷却后进入四极杆 质量过滤器进行选质, 然后进入线性离子阱反应器中 与冷却气 $\mathrm{He}$ 碰撞冷却至室温, 之后与 $\mathrm{CO}_{2}$ 分子反应. 反应一段时间后得到一系列产物离子，随后进入飞行 时间质谱进行检测. 在之前的工作中，已经证明团簇 离子在反应之前已接近室温 ${ }^{[51]}$. 参考文献 [51]中详细 描述了计算反应速率常数的方法.

\section{2 计算方法}

所有密度泛函理论(density functional theory, DFT) 的计算均使用Gaussian 09程序包 ${ }^{[52]}$, 使用BLYP ${ }^{[33,54]}$ 交 换-相关泛函和6-311+G(d)基组 ${ }^{[55,56]}$. 在14种测试方法 中 BLYP和B $1 \mathrm{~B} 95$ 均可很好地预测 $\mathrm{Cr}-\mathrm{Cr} 、 \mathrm{Cr}-\mathrm{N}$ 、 $\mathrm{Cr}-\mathrm{O} 、 \mathrm{C}-\mathrm{O}$ 和 $\mathrm{N}-\mathrm{O}$ 键的键解离能 (表 $\mathrm{S} 1$, 附加材料), 但 BLYP能够计算出更准确的 $\mathrm{Cr}-\mathrm{Cr}$ 键能，因此本文采用 BLYP方法. 其他含铬配合物也采用相同的方法获得 了可信的结果 ${ }^{[57,58]}$. 为了探究不同基组的影响, 我们 测试了较大的基组, 即对铬原子使用def2-QZVP ${ }^{[59]}$, 对 $\mathrm{N} 、 \mathrm{C} 、 \mathrm{O}$ 原子使用 aug-cc-pVTZ ${ }^{[60]}$ 。利用BLYP/def2QZVP和aug-cc-pVTZ计算的 $\mathrm{Cr}_{2} \mathrm{~N}^{-}$与 $\mathrm{CO}_{2}$ 反应的势能 面(potential energy surface, PES)与使用6-311+G(d)基 组计算的PES非常相似(图S1, 附加材料), 因此本文使 用6-311+G(d)基组. 反应机理计算涉及反应中间体(intermediate, IM)和过渡态(transition state, TS)的几何优 化. 用振动频率计算以检查IM或TS的频率中是否为没 有或仅有一个虚频. 内坐标(intrinsic reaction coordinate, IRC) 计算 ${ }^{[61,62]}$ 被用于确定得到的过渡态是否连 接两边的稳定中间体. 给出的能量 $\left(\Delta H_{0 \mathrm{~K}}, \mathrm{eV}\right)$ 已经经 过零点振动能校正. 使用NBO 6.0进行自然键轨道(natural bond orbital, NBO) 分析 ${ }^{[63]}$.

\section{3 结果与讨论}

$\mathrm{Cr}_{2} \mathrm{~N}^{-}$阴离子 $(m / z=118)$ 通过四极杆选质(图1(a)), 并在离子阱反应器中与 $\mathrm{CO}_{2}$ 分子反应(图1(b)). 如图 1 (b)所示，当 $\mathrm{Cr}_{2} \mathrm{~N}^{-}$阴离子与 $42 \mathrm{mPa} \mathrm{CO}_{2}$ 反应 $0.8 \mathrm{~ms}$ 时, 可以得到两种产物离子: $\mathrm{Cr}_{2} \mathrm{NO}^{-}$和 $\mathrm{Cr}_{2} \mathrm{NO}_{2}{ }^{-}$. 通过 $\mathrm{Cr}_{2} \mathrm{~N}^{-}$阴离子与 $\mathrm{CO}_{2}$ 反应气压力关系图可以看到(图2 (a)), $\mathrm{Cr}_{2} \mathrm{NO}^{-}$的相对离子强度并未随反应气体压力的 增加而显著增加，表明该团簇被其他反应迅速消耗. 基于最小二乘法拟合过程，我们可以得出结论： 

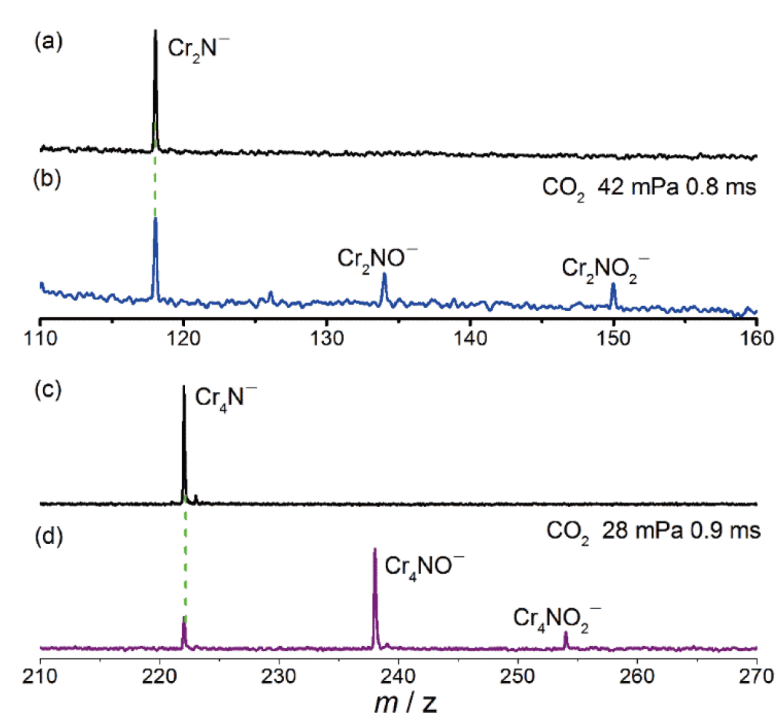

图 1 选质的 $\mathrm{Cr}_{2} \mathrm{~N}^{-}(\mathrm{a})$ 和 $\mathrm{Cr}_{4} \mathrm{~N}^{-}$(c) 分别与 $\mathrm{CO}_{2}$ 反应 $(\mathrm{b}, \mathrm{d})$ 的飞 行时间质谱图. 反应时间以及离子阱反应器中 $\mathrm{CO}_{2}$ 的有效压 力已给出(网络版彩图)

Figure 1 Time-of-flight mass spectra for the reactions of massselected $\mathrm{Cr}_{2} \mathrm{~N}^{-}$(a) with $\mathrm{CO}_{2}$ (b) for $0.8 \mathrm{~ms}$, and $\mathrm{Cr}_{4} \mathrm{~N}^{-}$(c) with $\mathrm{CO}_{2}$ (d) for $0.9 \mathrm{~ms}$. The effective reactant gas pressures are shown (color online).

$\mathrm{Cr}_{2} \mathrm{NO}_{2}{ }^{-}$的形成是由于 $\mathrm{Cr}_{2} \mathrm{NO}^{-}$与第二个 $\mathrm{CO}_{2}$ 分子继续 反应得到. 观察到的反应如下:

$$
\begin{aligned}
& \mathrm{Cr}_{2} \mathrm{~N}^{-}+\mathrm{CO}_{2} \rightarrow \mathrm{Cr}_{2} \mathrm{NO}^{-}+\mathrm{CO} \\
& \mathrm{Cr}_{2} \mathrm{NO}^{-}+\mathrm{CO}_{2} \rightarrow \mathrm{Cr}_{2} \mathrm{NO}_{2}{ }^{-}+\mathrm{CO}
\end{aligned}
$$

线性离子阱中准一级反应速率常数是确定的. 通 过最小二乘法拟合估算反应(1)和(2)的反应速率 $k_{1}$ 分 别为 $(6.4 \pm 0.3) \times 10^{-11}$ 和 $(1.3 \pm 0.3) \times 10^{-10} \mathrm{~cm}^{3} /($ molecule $\mathrm{s}$ ), 反应效率 $(\Phi)$ 分别为 $9 \%$ 和 $19 \%{ }^{[64]}$. 此外, $\mathrm{Cr}_{2} \mathrm{NH}^{-}$阴离子 团簇也可以活化 $\mathrm{CO}_{2}$ 的 $\mathrm{C}-\mathrm{O}$ 键生成 $\mathrm{Cr}_{2} \mathrm{NHO}^{-}$和 $\mathrm{Cr}_{2} \mathrm{NHO}_{2}^{-}$(图S2(a)). 但在反应达到平衡时, 第二个反 应的产物 $\mathrm{Cr}_{2} \mathrm{NO}_{2}{ }^{-}$是 $\mathrm{Cr}_{2} \mathrm{~N}^{-} / \mathrm{CO}_{2}$ 反应体系的主要产物, 而 $\mathrm{Cr}_{2} \mathrm{NH}^{-} / \mathrm{CO}_{2}$ 反应中第一个反应的产物 $\mathrm{Cr}_{2} \mathrm{NHO}^{-}$是 主要产物. 值得注意的是, 本文中所研究的 $\mathrm{Cr}_{x} \mathrm{~N}^{-}$ $(x=2 \sim 7)$ 阴离子团簇均可与 $\mathrm{CO}_{2}$ 反应生成产物峰 $\mathrm{Cr}_{x} \mathrm{NO}^{-}$和 $\mathrm{Cr}_{x} \mathrm{NO}_{2}^{-}$, 并且 $\mathrm{Cr}_{x} \mathrm{NO}^{-}$的相对离子强度不会 随反应气体压力的增加而显著增加，表明 $\mathrm{Cr}_{x} \mathrm{NO}^{-}$是中 间产物, 可以被后续反应继续消耗(图S3). 如图2(b)所 示, 随着团簇尺寸的增加, $\mathrm{Cr}_{x} \mathrm{~N}^{-}(x=2 \sim 7)$ 与 $\mathrm{CO}_{2}$ 的反应 中次级反应的反应速率一直小于主反应的速率, 并且 该类团簇与 $\mathrm{CO}_{2}$ 的反应效率并没有明显降低, 我们推
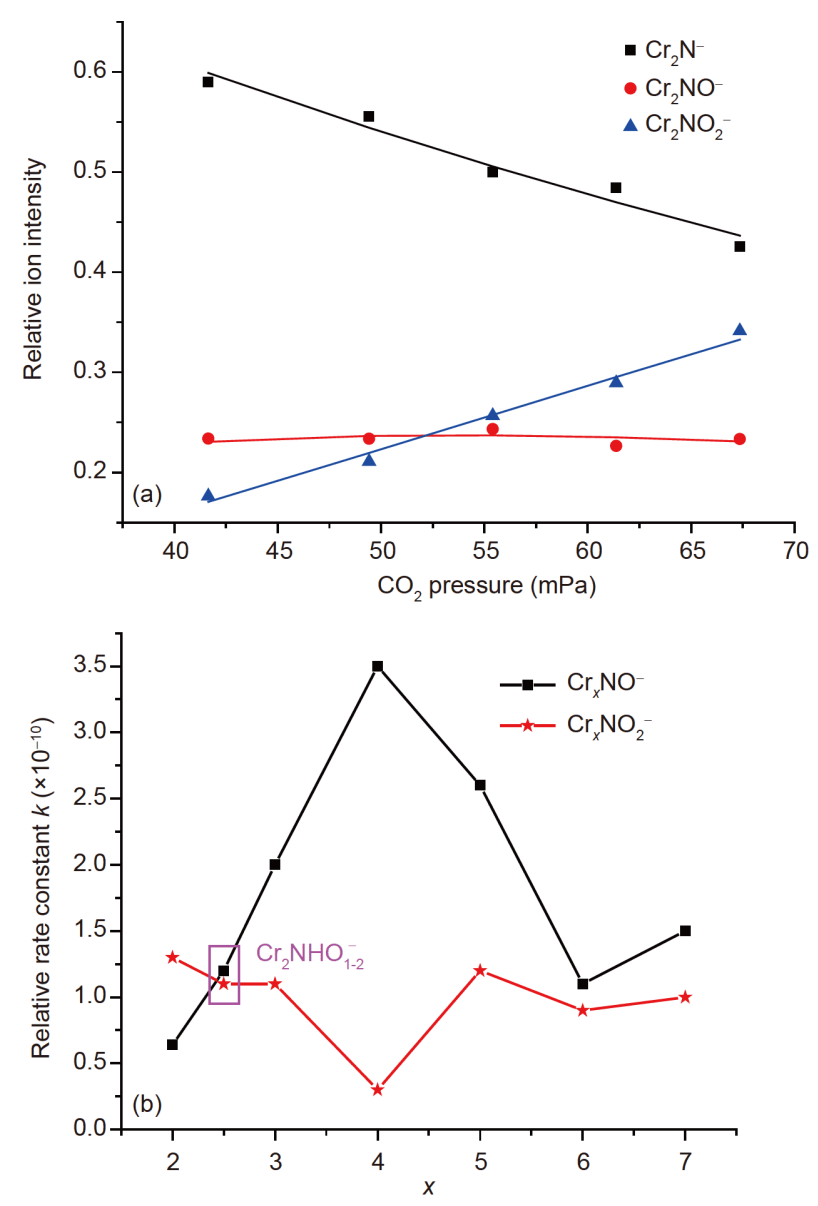

图 2 (a) $\mathrm{Cr}_{2} \mathrm{~N}^{-}$阴离子团簇与 $\mathrm{CO}_{2}$ 的反应速率拟合图, 反应 时间为 $0.8 \mathrm{~ms}$; (b) $\mathrm{Cr}_{x} \mathrm{~N}^{-}$阴离子团簇与 $\mathrm{CO}_{2}$ 反应 (表 $\mathrm{S} 2$ ) 的绝对 速率常数 $\left(k_{1}\right) . x$ 表示铬原子的数目(网络版彩图)

Figure 2 (a) Variation of the relative intensities of the reactant and product cluster ions in the reaction between $\mathrm{Cr}_{2} \mathrm{~N}^{-}$and $\mathrm{CO}_{2}$ with respect to the experimental reactant gas pressure. The reaction time is $0.8 \mathrm{~ms}$. (b) Absolute rate constants $\left(k_{1}\right)$ of $\mathrm{Cr}_{x} \mathrm{~N}^{-}$with $\mathrm{CO}_{2} . x$ represents the number of $\mathrm{Cr}$ atoms (color online).

断铬氮阴离子团簇在纳米尺寸可能仍能保留其高的反 应活性. 在研究的尺寸范围内, 团簇 $\mathrm{Cr}_{4} \mathrm{~N}^{-}$和 $\mathrm{Cr}_{5} \mathrm{~N}^{-}$对 $\mathrm{CO}_{2}$ 具有较高的反应活性.

为了获得 $\mathrm{Cr}_{2 \sim 4} \mathrm{~N}^{-}$阴离子团簇的结构及其与 $\mathrm{CO}_{2}$ 的 反应机理, 我们进行了DFT计算. 如图3 和图S4所示, $\mathrm{Cr}_{2} \mathrm{~N}^{-}$阴离子团簇的电子态为七重态, 所属点群为 $D_{\infty h}$, $\mathrm{N}$ 原子与两个 $\mathrm{Cr}$ 原子成键. 在八重态的 $\mathrm{Cr}_{2} \mathrm{NH}^{-}$团簇中, 一个 $\mathrm{H}$ 原子与一个 $\mathrm{Cr}$ 原子结合, $\mathrm{Cr}-\mathrm{N}-\mathrm{Cr}$ 结合呈线性. 五重态的 $\mathrm{Cr}_{3} \mathrm{~N}^{-}$为 $\left(C_{2 v}\right)$ 平面菱形结构, 存在直接相连的 $\mathrm{Cr}-\mathrm{Cr}$ 键 (三重态的 $\mathrm{Cr}_{3} \mathrm{~N}^{-}$能量也较低). $\mathrm{Cr}_{4} \mathrm{~N}^{-}$阴离子团 簇的最稳定构型呈 “ $\Lambda$ ”形, 点群为 $C_{2 v}$, 四个 $\mathrm{Cr}$ 原子形成 


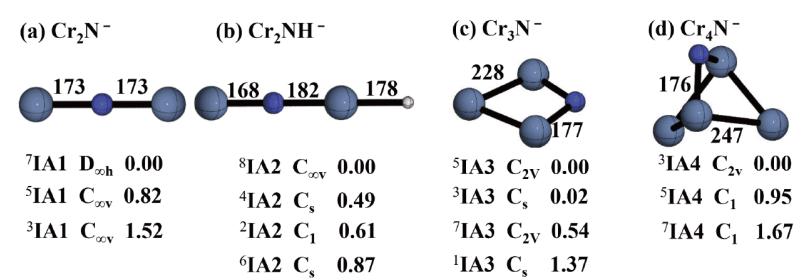

图 3 DFT计算的 $\mathrm{Cr}_{2} \mathrm{~N}^{-}$(a)、 $\mathrm{Cr}_{2} \mathrm{NH}^{-}$(b)、 $\mathrm{Cr}_{3} \mathrm{~N}^{-}$(c) 和 $\mathrm{Cr}_{4} \mathrm{~N}^{-}$ (d)的结构. 在每个结构下都给出了点群和自旋多重度. 上标 指示自旋多重度, 一些键长已给出(单位: pm) (网络版彩图)

Figure 3 DFT-calculated structures of $\mathrm{Cr}_{2} \mathrm{~N}^{-}$(a), $\mathrm{Cr}_{2} \mathrm{NH}^{-}$(b), $\mathrm{Cr}_{3} \mathrm{~N}^{-}$ (c) and $\mathrm{Cr}_{4} \mathrm{~N}^{-}$(d). The relative energies $\left(\Delta H_{0 \mathrm{~K}}\right.$, in $\left.\mathrm{eV}\right)$ with respect to the lowest-lying isomer are given. The point group and spin states (the superscripts) are given under each structure. Some bond lengths are given in pm (color online).

一个四元环，其中两个 $\mathrm{Cr}$ 原子与 $\mathrm{N}$ 原子键合. 目前，我 们没有进一步研究其他较大的 $\mathrm{Cr}_{x} \mathrm{~N}^{-}(x \geq 5)$ 团簇结构, 但预计会出现非平面几何构型和低自旋多重度. NBO 分析表明, $\mathrm{Cr}_{x} \mathrm{~N}^{-}$中 $\mathrm{Cr}-\mathrm{Cr}$ 和 $\mathrm{Cr}-\mathrm{N}$ 键的Wiberg键指数 (Wiberg bond index, WBI)均为 1 , 负电荷主要位于 $\mathrm{N}$ 原 子上. 文献报道中有关于 $\mathrm{Cr}_{x}$ 的构型 ${ }^{[65]}$ 及 $\mathrm{Cr}_{x}{ }^{-}$的光电子 能谱研究. 根据王来生等 ${ }^{[65]}$ 的报道, $\mathrm{Cr}_{2 \sim 4}$ 均为平面构 型. $\mathrm{Cr}_{2 \sim 3} \mathrm{~N}^{-}$阴离子的构型是平面, 而 $\mathrm{Cr}_{4} \mathrm{~N}^{-}$的构型已经 是立体构型. 此外, 光电子能谱实验结果表明, $\mathrm{Cr}_{x}{ }^{-}$ $(x=2 、 3 、 4)$ 的绝热解离能 (adiabatic detachment energy, $\mathrm{ADE}$ )分别为 $0.5 、 1.4$ 和 $1.3 \mathrm{eV}^{[66]}$. 基于本论文中 所采用的泛函和基组, DFT计算获得的 $\mathrm{Cr}_{x}{ }^{-}(x=2 、 3$ 、

4)的 $\mathrm{ADE}$ 分别为 $0.40 、 1.66$ 和 $1.22 \mathrm{eV}$, 与实验报道值基 本吻合 ${ }^{[66]}$, 我们算得的 $\mathrm{Cr}_{3}{ }^{-}$最稳定构型为 3 个 $\mathrm{Cr}$ 原子呈 直线型排布且自旋多重度为 6 . 三角形构型的 $\mathrm{Cr}_{3}{ }^{-}$的 $\mathrm{ADE}$ 为 $0.60 \mathrm{eV} . \mathrm{Cr}_{x} \mathrm{~N}^{-}(x=2 、 3 、 4)$ 的 $\mathrm{ADE}$ 数值分别为 1.92、0.73和 $1.64 \mathrm{eV}$, 说明在 $\mathrm{Cr}_{x}{ }^{-}$中引入一个 $\mathrm{N}$ 原子可 以显著改变团簇的电子结构. 此外, 在团簇源中可能 还存在少量 $\mathrm{Cr}_{x} \mathrm{~N}^{-}$阴离子的低能量异构体, 在现有的研 究条件下无法排除这些异构体存在的可能性. 光电子 能谱是表征阴离子结构的有效手段, 但是 $\mathrm{Cr}_{2 \sim 4} \mathrm{~N}^{-}$团簇 产生强度较低, 难以开展相关实验.

$\mathrm{Cr}_{2} \mathrm{~N}^{-}$与 $\mathrm{CO}_{2}$ 连续反应(反应(1)和(2))的势能面(potential energy surface, PES)如图4所示. $\mathrm{CO}_{2}$ 分子可以直 接吸附在 $\mathrm{Cr}_{2} \mathrm{~N}^{-}$阴离子上形成中间体 I1，其中 $\mathrm{CO}_{2}$ 分子 中的一个 $\mathrm{C}$ 原子和一个 $\mathrm{O}$ 原子与 $\mathrm{Cr}_{2} \mathrm{~N}^{-}$中的一个 $\mathrm{Cr}$ 原子 相连; $\mathrm{CO}_{2}$ 中键角 $\angle \mathrm{O}-\mathrm{C}-\mathrm{O}$ 的度数从 $\mathrm{CO}_{2}$ 分子中的 $180^{\circ}$ 变到 $\mathbf{I}$ 中的 $125^{\circ}$, 表明 $\mathrm{CO}_{2}$ 被活化. 根据 $\mathrm{NBO}$ 分析, 约 $-0.86 e$ 的电子转移到 $\mathrm{CO}_{2}$ 部分上. 在 $\left(\mathrm{TiO}_{2}\right)_{n} \mathrm{O}^{-} / \mathrm{CO}_{2}{ }^{[67]}$
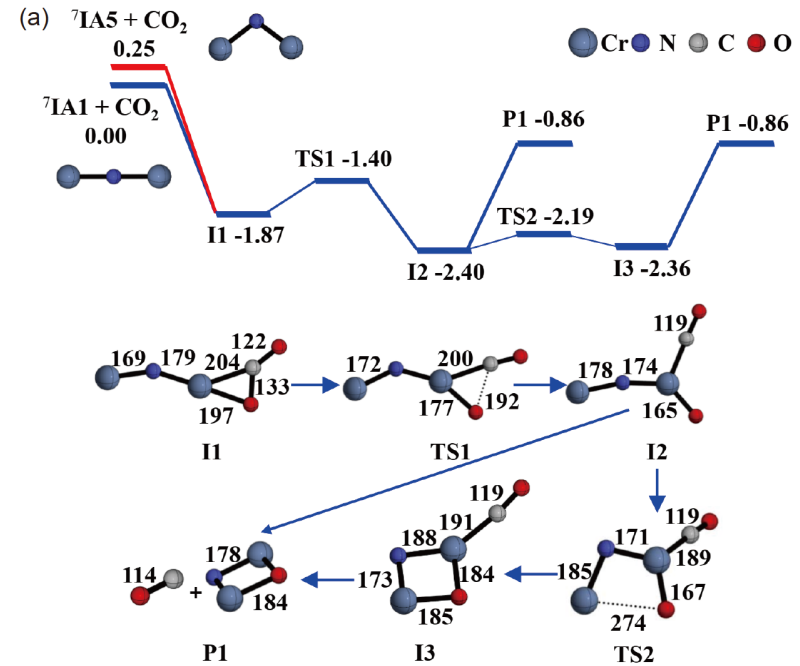

(b)

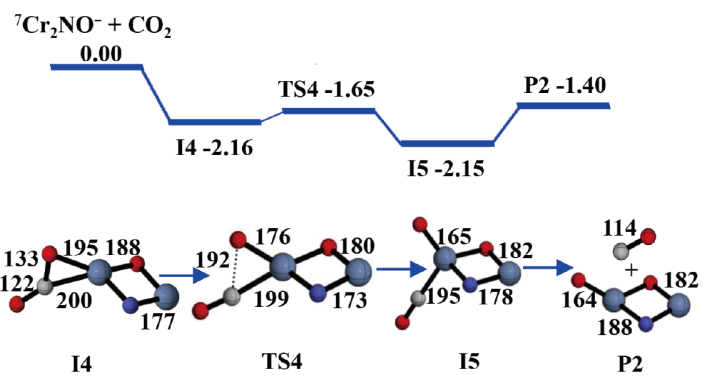

图 4 DFT计算的 $\mathrm{Cr}_{2} \mathrm{~N}^{-}\left({ }^{7}\right.$ IA1, ${ }^{7}$ IA5) (a) 和 $\mathrm{Cr}_{2} \mathrm{NO}^{-}$( ${ }^{7}$ IA21) (b) 与 $\mathrm{CO}_{2}$ 反应的势能面曲线. 其中一些键长和键角已给出. 反应物、过渡态和中间体的能量均给出, 所示能量均以反应 物能量为零点, 并进行了零点振动能校正 $\left(\Delta H_{0 \mathrm{~K}}, \mathrm{eV}\right.$; 键长单 位: pm) (网络版彩图)

Figure 4 DFT-calculated potential energy surfaces for the reactions of $\mathrm{Cr}_{2} \mathrm{~N}^{-}$with $\mathrm{CO}_{2}$ (a), and $\mathrm{Cr}_{2} \mathrm{NO}^{-}$with $\mathrm{CO}_{2}$ (b). Some bond lengths and bond angles are given in pm and in degree, respectively. The zero-point vibration corrected energies $\left(\Delta H_{0 \mathrm{~K}}\right.$ in $\left.\mathrm{eV}\right)$ of the reaction intermediates, transition states, and products with respect to the separated reactants are given (color online).

和 $\mathrm{FeH}^{-} / \mathrm{CO}_{2}{ }^{[47]}$ 体系中也观察到类似现象. 在越过过渡 态TS1后发生氧原子转移过程, 生成中间体 $\mathrm{Cr}_{2} \mathrm{NOCO}^{-}$ (I2). $\mathrm{CO}$ 单元从I3中脱离后该反应完成, $\mathrm{CO}$ 单元也可 以从 $\mathbf{I 2}$ 直接脱附生成P1. 对于反应(2), 新生成的 $\mathrm{Cr}_{2} \mathrm{NO}^{-}$可以进一步与另外一个 $\mathrm{CO}_{2}$ 分子反应, 从而导 致 $\mathrm{Cr}_{2} \mathrm{NO}_{2}{ }^{-}$和 $\mathrm{CO}$ 的形成. 如图4(b) 所示, $\mathrm{CO}_{2}$ 吸附在 $\mathrm{Cr}_{2} \mathrm{NO}^{-}$上之后, 一个O原子从 $\mathrm{CO}_{2}$ 中抽离出来到 $\mathrm{Cr}_{2} \mathrm{NO}^{-}$ $(\mathbf{I 4} \rightarrow \mathbf{T S} 4 \rightarrow \mathbf{I 5})$ 形成带有 $\mathrm{Cr}-\mathrm{O}-\mathrm{Cr}-\mathrm{N}$ 环的 $\mathrm{Cr}_{2} \mathrm{NO}_{2}{ }^{-}$. 随 着 $\mathrm{Cr}_{2} \mathrm{NO}_{2}{ }^{-}$的生成, 新形成的 $\mathrm{CO}$ 单元可以直接从中间 体I5释放出来. 利用Rice-Ramsperger-Kassel-Marcus (RRKM) 理论 ${ }^{[68]}$ 和变分过渡态理论(variational transition state theory, VTST ${ }^{[69]}$ 可以估算通过过渡态TS1形 
成 CO单元 $(\mathbf{I 1} \rightarrow \mathbf{T S} 1$, 图 $4(\mathrm{a}))$ 的速率 $\left(k_{\mathrm{conversion}}\right)$ 和 $\mathrm{CO}$ 解 离 $\left(\mathbf{I 3} \rightarrow \mathbf{P 1}\right.$, 图4(a))速率 $\left(k_{\mathrm{d}}\right)$. 由表1可知, 对于反应(1), $k_{\mathrm{d}}\left(2.1 \times 10^{11} \mathrm{~s}^{-1}\right)$ 小于 $k_{\text {conversion }}\left(8.1 \times 10^{11} \mathrm{~s}^{-1}\right)$. 在反应 $(2)$ 中, 释放 $\mathrm{CO}$ 的 $k_{\mathrm{d}}$ 和 $k_{\text {conversion }}(\mathrm{I4} \rightarrow \mathrm{TS} 4)$ 分别为 $2.4 \times 10^{12}$ 和 $8.7 \times 10^{11} \mathrm{~s}^{-1}$. 因此, 释放 $\mathrm{CO}$ 是决定速率的步骤. 值得注 意的是, $k_{\text {conversion }}$ 和 $k_{\mathrm{d}}$ 的数值是基于DFT计算结果, 因此 可能存在一定的偏差.

由于 $\mathrm{Cr}_{2} \mathrm{NH}^{-}$比 $\mathrm{Cr}_{2} \mathrm{~N}^{-}$多一个 $\mathrm{H}$ 原子, 并且在所研究 的 $\mathrm{Cr}_{x} \mathrm{~N}^{-}$体系中 $\mathrm{Cr}_{4} \mathrm{~N}^{-}$对 $\mathrm{CO}_{2}$ 的反应活性最高, 因此我 们进一步计算了 $\mathrm{Cr}_{2} \mathrm{NH}^{-}$和 $\mathrm{Cr}_{4} \mathrm{~N}^{-}$与 $\mathrm{CO}_{2}$ 的反应势能面 (图S5 和S6). 这些反应势能面与图4所示的过程类似. 根据DFT的计算结果以及 $\mathrm{CO}$ 脱附的速率 $\left(k_{\mathrm{d}}\right)$ 和表 1 所 示中间体克服能垒的转化速率 $k_{\text {conversion, }}$ 发现所研究的 反应具有以下几个共同特征: (1) 在 $\mathrm{CO}_{2}$ 与团簇反应形 成第一个中间体时, $\mathrm{CO}_{2}$ 中有一个氧原子与 $\mathrm{Cr}_{x} \mathrm{~N}^{-}$中的 $\mathrm{Cr}$ 原子配位, 释放足够的能量 $(>1.5 \mathrm{eV})$. 由于电子从 $\mathrm{Cr}_{x} \mathrm{~N}^{-}$转移至被吸附的 $\mathrm{CO}_{2}$ 单元中, $\mathrm{O}-\mathrm{C}-\mathrm{O}$ 构型变为弯 曲. (2) 除了 $\mathrm{Cr}_{4} \mathrm{NO}^{-}$与 $\mathrm{CO}_{2}$ 反应路径中的第一个过渡态 外, $\mathrm{O}$ 原子转移过程的活化能相似(约 $0.5 \mathrm{eV}$ ). (3) 在所 研究的连续反应的第一个 $\mathrm{CO}_{2}$ 分子还原反应中, 生成 的 $\mathrm{CO}$ 单元从 $\left[\mathrm{Cr}_{x} \mathrm{NO}^{-} \cdots \mathrm{CO}\right]$ 中间体中离去是可能的决 速步骤; 对于第二个 $\mathrm{CO}_{2}$ 分子还原反应, 越过过渡态的 转化步骤是限速步骤.

当前实验结果表明, $\mathrm{Cr}_{4} \mathrm{~N}^{-} / \mathrm{CO}_{2}$ 的反应速率是 $k_{1}$ $\left(\mathrm{Cr}_{2} \mathrm{~N}^{-}+\mathrm{CO}_{2}\right)$ 的2倍. 但是目前计算得到的反应势能面 和解离速率(表1)等表明应该还有其他影响因素. 进一 步的理论计算表明, $\mathrm{Cr}_{4} \mathrm{~N}^{-} / \mathrm{CO}_{2}$ 和 $\mathrm{Cr}_{4} \mathrm{NO}^{-} / \mathrm{CO}_{2}$ 体系中 新形成的 $\mathrm{CO}$ 单元可以很容易在多个 $\mathrm{Cr}$ 原子上转移, 从
而可以从多个金属铬位点被释放 $\left(\mathbf{I 1 3}, \mathbf{I 1 4} \rightarrow{ }^{3} \mathrm{P5} ;{ }^{7} \mathbf{I 1}\right.$, ${ }^{7} \mathrm{I18} \rightarrow{ }^{7} \mathbf{P 6}$, 图S6). 此外, 通过分析DFT计算的 $\mathrm{Cr}_{2} \mathrm{~N}^{-}$和 $\mathrm{Cr}_{4} \mathrm{~N}^{-}$的结构也可以发现, $\mathrm{Cr}_{4} \mathrm{~N}^{-}$的活性位点也比 $\mathrm{Cr}_{2}$ $\mathrm{N}^{-}$多, 这可能是 $\mathrm{Cr}_{4} \mathrm{~N}^{-}$比 $\mathrm{Cr}_{2} \mathrm{~N}^{-}$反应速率快的另一个 原因.

随着 $\mathrm{Cr}$ 原子数 $x$ 的增加, $\mathrm{Cr}_{x} \mathrm{~N}^{-}$阴离子中 $\mathrm{Cr}$ 的配位 数也在增加(表 $\mathrm{S} 3$ ). 对比 $\mathrm{Cr}_{x} \mathrm{~N}^{-} 、 \mathrm{Cr}_{x} \mathrm{NO}^{-}$和 $\mathrm{Cr}_{x} \mathrm{NO}_{2}{ }^{-}$的 结构可以发现, $\mathrm{Cr}_{x} \mathrm{NO}^{-}$和 $\mathrm{Cr}_{x} \mathrm{NO}_{2}^{-}(x=2 、 4)$ 中的 $\mathrm{O}$ 原子 是以桥氧键结合, 而非端氧键结合的方式. 对于较大尺 寸的 $\mathrm{Cr}_{x} \mathrm{~N}^{-}(x>7)$ 团簇, 对 $\mathrm{CO}_{2}$ 的反应性也可能持续存 在, 因此需要后续进一步研究较大尺寸的 $\mathrm{Cr}_{x} \mathrm{~N}^{-}$团簇 (如纳米级阴离子团簇) 的反应性. 但是随着 $\mathrm{Cr}$ 原子数 量的增加, 预计团簇的立体几何构型会导致 $\mathrm{Cr}$ 原子的 配位数增高. 尺寸的增大也意味着更多的 $\mathrm{CO}$ 分子解吸 位点, 这也有利于 $\mathrm{CO}$ 的释放. 因此, 合成相对较大的 铬-氮活性位点可能是促进 $\mathrm{CO}$ 释放的一种方法. 此外, 在 $\mathrm{Cr}_{x} \mathrm{~N}^{-}$与 $\mathrm{CO}_{2}$ 的反应中未观察到 $\mathrm{CO}_{2}$ 的吸附产物, 这 表明在负载氮化铬催化剂上可能不会发生由于催化活 性位被 $\mathrm{CO}_{2}$ 吸附占据而引起的催化剂失活.

\section{4 结论}

在这项工作中, 我们利用实验和理论计算方法研 究了 $\mathrm{Cr}_{x} \mathrm{~N}^{-}(x=2 \sim 7)$ 和 $\mathrm{Cr}_{2} \mathrm{NH}^{-}$阴离子与 $\mathrm{CO}_{2}$ 分子的反 应, 发现了存在的反应通道: $\mathrm{Cr}_{x} \mathrm{~N}^{-}$能够连续活化两个 $\mathrm{CO}_{2}$ 分子, 生成 $\mathrm{Cr}_{x} \mathrm{NO}^{-}$和 $\mathrm{Cr}_{x} \mathrm{NO}_{2}^{-}$并释放两个 $\mathrm{CO}$ 分子. 之前文献中并未有关于气相过渡金属氮化物团簇阴离 子活化 $\mathrm{CO}_{2}$ 的报道. 此外, 在所研究的反应中没有观察

表 1 RRKM理论和VTST估算中间体克服过渡态形成CO单元的绝对速率常数 $\left(k_{\text {conversion }}\right)$ 和CO单元的解离速率 $\left(k_{\mathrm{d}}\right)^{\text {a) }}$

Table 1 The calculated VTST rates of CO desorption into products $\left(k_{\mathrm{d}}, \mathrm{s}^{-1}\right)$ and the RRKM rates of traversing the highest TSs from intermediates $\left(k_{\text {conversion }}, \mathrm{s}^{-1}\right)$

\begin{tabular}{|c|c|c|c|c|}
\hline \multirow{2}{*}{ 反应 } & \multicolumn{2}{|c|}{$k_{\mathrm{d}}$} & \multicolumn{2}{|c|}{$k_{\text {conversion }}$} \\
\hline & 反应步骤 & $k_{\mathrm{d}}$ & 反应步骤 & $k_{\text {conversion }}$ \\
\hline $\mathrm{Cr}_{2} \mathrm{~N}^{-}+\mathrm{CO}_{2}$ & ${ }^{7} \mathbf{I 3} \rightarrow{ }^{7} \mathbf{P} 1$ & $2.1 \times 10^{11}$ & ${ }^{7} \mathrm{I} 1 \rightarrow{ }^{7} \mathrm{TS} 1 \rightarrow{ }^{7} \mathrm{I} 2$ & $8.1 \times 10^{11}$ \\
\hline $\mathrm{Cr}_{2} \mathrm{NO}^{-}+\mathrm{CO}_{2}$ & ${ }^{7} \mathrm{I} 5 \rightarrow{ }^{7} \mathrm{P} 2$ & $2.4 \times 10^{12}$ & ${ }^{7} \mathrm{I} 4 \rightarrow{ }^{7} \mathrm{TS} 4 \rightarrow{ }^{7} \mathrm{I5}$ & $8.7 \times 10^{11}$ \\
\hline $\mathrm{Cr}_{2} \mathrm{NH}^{-}+\mathrm{CO}_{2}$ & ${ }^{8} \mathrm{I} 8 \rightarrow{ }^{8} \mathrm{P3}$ & $2.5 \times 10^{11}$ & ${ }^{8} \mathbf{I} \mathbf{I 6} \rightarrow{ }^{8} \mathrm{TS6} \rightarrow{ }^{8} \mathbf{I} 7$ & $1.4 \times 10^{11}$ \\
\hline $\mathrm{Cr}_{2} \mathrm{NHO}^{-}+\mathrm{CO}_{2}$ & ${ }^{6} \mathbf{I 1 0} \rightarrow{ }^{6} \mathbf{P 4}$ & $2.3 \times 10^{12}$ & ${ }^{6} \mathbf{I 9} \rightarrow{ }^{6} \mathbf{T S} 9 \rightarrow{ }^{6} \mathbf{I} 10$ & $2.9 \times 10^{11}$ \\
\hline $\mathrm{Cr}_{4} \mathrm{~N}^{-}+\mathrm{CO}_{2}$ & ${ }^{3} \mathrm{I} 13 \rightarrow{ }^{3} \mathrm{P} 5$ & $2.6 \times 10^{10}$ & ${ }^{3} \mathrm{I} 11 \rightarrow{ }^{3} \mathrm{TS} 11 \rightarrow{ }^{3} \mathrm{I} 12$ & $3.1 \times 10^{11}$ \\
\hline $\mathrm{Cr}_{4} \mathrm{NO}^{-}+\mathrm{CO}_{2}$ & ${ }^{7} \mathrm{I} 17 \rightarrow{ }^{7} \mathrm{P} 6$ & $4.1 \times 10^{11}$ & ${ }^{3} \mathrm{I} 15 \rightarrow{ }^{3} \mathrm{TS} 15 \rightarrow{ }^{3} \mathrm{I} 16$ & $1.5 \times 10^{10}$ \\
\hline
\end{tabular}

a) $\mathrm{Cr}_{2} \mathrm{NH}^{-} 、 \mathrm{Cr}_{2} \mathrm{NHO}^{-} 、 \mathrm{Cr}_{4} \mathrm{~N}^{-}$和 $\mathrm{Cr}_{4} \mathrm{NO}^{-}$与 $\mathrm{CO}_{2}$ 的反应步骤见图 $\mathrm{S} 5$ 和 $\mathrm{S} 6$ 
到 $\mathrm{CO}_{2}$ 吸附产物. 与其他已报道的活性团簇相比, $\mathrm{Cr}_{x} \mathrm{~N}^{-} \quad(x=2 \sim 7)$ 阴离子对 $\mathrm{CO}_{2}$ 具有很高的反应性和 增加到4时，团簇构型从平面结构变为三维结构，并阐 $100 \%$ 的选择性. 详细的机理分析表明, 当铬原子数从 2 述了相关的连续反应机理. 这些结果背后的基本机理 有助于设计高效的 $\mathrm{CO}_{2}$ 还原催化剂.

\section{补充材料}

本文的补充材料见网络版http://chemen.scichina.com. 补充材料为作者提供的原始数据, 作者对其学术质量和内容负责.

\section{参考文献}

1 Aresta M, Dibenedetto A, Angelini A. Chem Rev, 2014, 114: 1709-1742

2 Aziz MAA, Jalil AA, Triwahyono S, Ahmad A. Green Chem, 2015, 17: 2647-2663

3 Wang X, Shi H, Szanyi J. Nat Commun, 2017, 8: 513

4 Kwak JH, Kovarik L, Szanyi J. ACS Catal, 2013, 3: 2094-2100

5 Sun F, Yan C, Wang Z, Guo C, Huang S. Int J Hydrogen Energy, 2015, 40: 15985-15993

6 Prairie M. J Catal, 1991, 129: 130-144

7 Wang W, Wang S, Ma X, Gong J. Chem Soc Rev, 2011, 40: 3703-3727

8 Zhang X, Zhu X, Lin L, Yao S, Zhang M, Liu X, Wang X, Li YW, Shi C, Ma D. ACS Catal, 2017, 7: 912-918

9 Schwarz H. Coord Chem Rev, 2016, 334: 112-123

10 Schwarz H. Catal Sci Technol, 2017, 7: 4302-4314

11 Beyer MK, Berg CB, Bondybey VE. Phys Chem Chem Phys, 2001, 3: 1840-1847

12 Justes DR, Castleman Jr AW, Mitric R, Bonačić-Koutecký V. Eur Phys J, 2003, 24: 331-334

13 Johnson GE, Mitrić R, Bonačić-Koutecký V, Castleman Jr AW. Chem Phys Lett, 2009, 475: 1-9

14 Roithova J, Schroder D. Chem Rev, 2010, 110: 1170-1211

Zhao YX, Wu XN, Ma JB, He SG, Ding XL. Phys Chem Chem Phys, 2011, 13: 1925-1938

Yin S, Bernstein ER. Int J Mass Spectrometry, 2012, 321-322: 49-65

Ding XL, Wu XN, Zhao YX, He SG. Acc Chem Res, 2012, 45: 382-390

Sakuma K, Miyajima K, Mafuné F. J Phys Chem A, 2013, 117: 3260-3265

Harris BL, Waters T, Khairallah GN, O'Hair RAJ. J Phys Chem A, 2013, 117: 1124-1135

Schwarz H. Isr J Chem, 2014, 54: 1413-1431

Liu QY, He SG. Chem J Chinese U, 2014, 35: 665-688

Schwarz H, González-Navarrete P, Li J, Schlangen M, Sun X, Weiske T, Zhou S. Organometallics, 2016, 36: 8-17

Schwarz H, Shaik S, Li J. J Am Chem Soc, 2017, 139: 17201-17212

Zhao YX, Liu QY, Zhang MQ, He SG. Dalton Trans, 2016, 45: 11471-11495

Koyanagi GK, Bohme DK. J Phys Chem A, 2006, 110: 1232-1241

Han DM, Dai GL, Chen H, Wang Y, Zhong AG, Lin CP, Chen D. Int J Quantum Chem, 2011, 111: 2898-2909

Ricks AM, Brathwaite AD, Duncan MA. J Phys Chem A, 2013, 117: 11490-11498

Sievers MR, Armentrout PB. Inorg Chem, 1999, 38: 397-402

Di Tommaso S, Marino T, Rondinelli F, Russo N, Toscano M. J Chem Theor Comput, 2007, 3: 811-815

Dheandhanoo S, Chatterjee BK, Johnsen R. J Chem Phys, 1985, 83: 3327-3329

Wesendrup R, Schwarz H. Angew Chem Int Ed Engl, 1995, 34: 2033-2035

Schwarz J, Schwarz H. Organometallics, 1994, 13: 1518-1520

Firouzbakht M, Rijs NJ, González-Navarrete P, Schlangen M, Kaupp M, Schwarz H. Chem Eur J, 2016, 22: 10581-10589

Sievers MR, Armentrout PB. J Chem Phys, 1995, 102: 754-762

Lam TW, Zhang H, Siu CK. J Phys Chem A, 2015, 119: 2780-2792 
Firouzbakht M, Rijs NJ, Schlangen M, Kaupp M, Schwarz H. Top Catal, 2018, 61: 575-584

Firouzbakht M, Schlangen M, Kaupp M, Schwarz H. J Catal, 2016, 343: 68-74

Demireva M, Armentrout PB. Top Catal, 2018, 61: 3-19

Wang YC, Yang X, Geng ZY, Liu ZY. Chem Phys Lett, 2006, 431: 39-44

Herman J, Foutch JD, Davico GE. J Phys Chem A, 2007, 111: 2461-2468

Koszinowski K, Schröder D, Schwarz H. J Phys Chem A, 2003, 107: 4999-5006

Lourenço C, Michelini MC, Marçalo J, Gibson JK, Oliveira MC. J Phys Chem A, 2012, 116: 12399-12405

Zhou S, Li J, Firouzbakht M, Schlangen M, Schwarz H. J Am Chem Soc, 2017, 139: 6169-6176

Li J, Geng C, Weiske T, Schwarz H. Angew Chem Int Ed, 2020, doi: 10.1002/anie.202001223

Tang SY, Rijs NJ, Li J, Schlangen M, Schwarz H. Chem Eur J, 2015, 21: 8483-8490

Zhuang J, Li ZH, Fan K, Zhou M. J Phys Chem A, 2012, 116: 3388-3395

Jiang LX, Zhao C, Li XN, Chen H, He SG. Angew Chem Int Ed, 2017, 56: 4067

Yuan Z, Li ZY, Zhou ZX, Liu QY, Zhao YX, He SG. J Phys Chem C, 2014, 118: 14967-14976

Wu XN, Xu B, Meng JH, He SG. Int J Mass Spectrometry, 2012, 310: 57-64

Yuan Z, Zhao YX, Li XN, He SG. Int J Mass Spectrometry, 2013, 354-355: 105-112

Zhao YX, Li ZY, Yuan Z, Li XN, He SG. Angew Chem Int Ed, 2014, 53: 9482-9486

Frisch MJ, Trucks GW, Schlegel HB, et al. Gaussian 09, Revision D.01. Wallingford CT: Gaussian, Inc., 2013

Becke AD. Phys Rev A, 1988, 38: 3098-3100

Lee C, Yang W, Parr RG. Phys Rev B, 1988, 37: 785-789

Dobbs KD, Hehre WJ. J Comput Chem, 1987, 8: 861-879

Francl MM, Pietro WJ, Hehre WJ, Binkley JS, Gordon MS, DeFrees DJ, Pople JA. J Chem Phys, 1982, 77: 3654-3665

Wu LC, Hsu CW, Chuang YC, Lee GH, Tsai YC, Wang Y. J Phys Chem A, 2011, 115: 12602-12615

Yang B, Xu H, Xu X, Zheng W. J Phys Chem A, 2018, 122: 9886-9893

Weigend F, Ahlrichs R. Phys Chem Chem Phys, 2005, 7: 3297-3305

Dunning Jr TH. J Chem Phys, 1989, 90: 1007-1023

Gonzalez C, Schlegel HB. J Chem Phys, 1989, 90: 2154-2161

Gonzalez C, Schlegel HB. J Phys Chem, 1990, 94: 5523-5527

Glendening ED, Badenhoop JK, Reed AE, Carpenter JE, Bohmann JA, Morales CM, Landis CR, Weinhold F. NBO 6.0. Madison, WI: Theoretical Chemistry Institute, University of Wisconsin, 2013. http://nbo6.chem.wisc.edu/

Gioumousis G, Stevenson DP. J Chem Phys, 1958, 29: 294-299

Cheng H, Wang LS. Phys Rev Lett, 1996, 77: 51-54

Wang LS, Wu H, Cheng H. Phys Rev B, 1997, 55: 12884-12887

Ma JB, Xu B, Meng JH, Wu XN, Ding XL, Li XN, He SG. J Am Chem Soc, 2013, 135: 2991-2998

Raghavachari K, Trucks GW, Pople JA, Head-Gordon M. Chem Phys Lett, 1989, 157: 479-483

Steinfeld JI, Francisco JS, Hase WL. Chemical Kinetics and Dynamics. New Jersey: Prentice-Hall, 1999. 313-314 


\title{
The study on the reaction of gas-phase $\mathrm{Cr}_{x} \mathrm{~N}^{-}(x=2-7)$ anion clusters with $\mathrm{CO}_{2}$
}

\author{
Ming Wang ${ }^{\dagger}$, Haiyan Zhou ${ }^{\dagger}$, Jiatong Cui, Chuanxin Sun, Jiabi Ma ${ }^{*}$ \\ Key Laboratory of Cluster Science of Ministry of Education, Beijing Key Laboratory of Photoelectronic/Electrophotonic Conversion Materials, School \\ of Chemistry and Chemical Engineering, Beijing Institute of Technology, Beijing 102488, China \\ $\dagger$ These authors contributed equally to this work. \\ *Corresponding author (email: majiabi@bit.edu.cn)
}

\begin{abstract}
Efficient reduction of $\mathrm{CO}_{2}$ to $\mathrm{CO}$ under room temperature is of great importance for $\mathrm{CO}_{2}$ utilization. For the first time, the $\mathrm{CO}_{2}$ reduction by multinuclear transition-mental nitride cluster anions $\mathrm{Cr}_{x} \mathrm{~N}^{-}(x=2-7)$ under thermalcollision conditions has been identified by applying mass spectrometry in conjunction with density functional theory (DFT) calculations. The $\mathrm{Cr}_{x} \mathrm{~N}^{-}(x=2-7)$ and $\mathrm{Cr}_{2} \mathrm{NH}^{-}$clusters can react with $\mathrm{CO}_{2}$ to generate $\mathrm{Cr}_{x} \mathrm{NO}^{-}$and $\mathrm{CO}$, and the intermediate ternary anions $\mathrm{Cr}_{x} \mathrm{NO}^{-}$can further reduce another $\mathrm{CO}_{2}$ to form $\mathrm{Cr}_{x} \mathrm{NO}_{2}{ }^{-}$and one $\mathrm{CO}$ molecules. All of these consecutive reactions are with high reactivity, and no $\mathrm{CO}_{2}$ adsorbate is formed. As the cluster size increases from $\mathrm{Cr}_{2} \mathrm{~N}^{-}$ to $\mathrm{Cr}_{7} \mathrm{~N}^{-}$, the cluster reactivity first increases and then decreases slightly. The DFT calculations predict the structures of these reactive anions and reaction mechanisms for the consecutive reactions. This work shades new light into the design of active sites on reverse water gas shift catalysts.
\end{abstract}

Keywords: gas-phase reactions, carbon dioxide, transition metal nitride clusters, mass spectrometer, computational quantum chemistry

doi: $10.1360 /$ SSC-2020-0061 